\title{
Caldas y el gran cometa de 1807
}

\author{
J. Gregorio Portilla
}

Observatorio Astronómico Nacional, Facultad de Ciencias, Sede Bogotá, Universidad Nacional de Colombia

\begin{abstract}
Resumen
Se analizan las observaciones que realizó Francisco José de Caldas del gran cometa de 1807. A partir de distintas observaciones realizadas por astrónomos europeos, así como del conocimiento de los elementos orbitales del cometa, se calculó su trayectoria y se comparó con lo que observó Caldas, en particular, sobre su observación del 26 de septiembre de 1807, la que incluye coordenadas. Se discuten algunos aspectos tales como la forma como calculó las coordenadas del cometa así como la descripción morfológica del mismo. Se infiere la existencia de algún fenómeno atmosférico que dificultó, para Caldas, la observación adecuada de la cola del cometa. (c) 2017. Acad. Colomb. Cienc. Ex. Fis. Nat.
\end{abstract}

Palabras clave: Caldas, F. J.; Cometa; Gran cometa de 1807.

Caldas and the great comet of 1807

\begin{abstract}
Observations of the great comet of 1807 carried out by Francisco José de Caldas are analyzed. Starting from astronomical observations obtained by European astronomers as well as the knowledge of the orbital elements, the trajectory of the comet is calculated and then is compared with those reported by Caldas, in particular that of the 26 of september of 1807 which includes coordinates. Items such as how Caldas calculated the coordinates along with morphological description of the object is discussed. We infer the existence of some type of atmospheric phenomena which hindered, for Caldas, the ideal observation of the tail. (c) 2017. Acad. Colomb. Cienc. Ex. Fis. Nat.
\end{abstract}

Key words: Caldas, F. J.; Comet; Great comet of 1807.

\section{Introducción}

Francisco José de Caldas (1768-1816), natural de Popayán, realizó estudios superiores en leyes entre 1788 y 1791 en el Colegio del Rosario de Santafé de Bogotá al cabo de los cuales obtuvo el título de Bachiller de Derecho y Filosofía. Probablemente por problemas de salud o insatisfacción, no continuó sus estudios, que le hubieran permitido conseguir el título de Licenciado y de Doctor en derecho y realizar una distinguida carrera como jurisconsulto. En su lugar optó por una vida de estudio en diversos aspectos de las ciencias naturales tales como astronomía, botánica, geografía, meteorología, etc. Dado el enorme resago que tenía el continente americano en materia científica con relación a Europa y, por ende, la ausencia total de programas académicos en tales tópicos (la mayoría de los cuales, en el país sólo vendrían a crearse casi siglo y medio después) la apropiación del conocimiento que hace Caldas de tales temas los hace de forma completamente autodidacta, asimilándolos de los libros que podía encargar y adquirir.

Algunas pistas sugieren que para 1792, esto es, inmediatamente después de sus estudios de derecho, y ya de regreso en Popayán, estaba asimilando conocimientos astronómicos. Entre 1793 y 1794 ejerció en Popayán varias labores relacionadas con su profesión pero se ve obligado a suspenderlas debido a su delicado estado de salud. Se sabe que para mediados de 1795 trabaja como buhonero (vendedor de ropa itinerante) y que al año siguiente hace un viaje a Santafé cuyo propósito, a parte del comercial, es adquirir libros e instrumentos científicos.

Sus avances en asimilar conocimientos astronómicos son notables entre 1796 y 1798. Construye gnomones y cuartos de círculo para determinar latitudes así como observa eclipses de Luna y Sol como también emersiones y ocultaciones de los satélites de Júpiter para determinar longitudes. Su experticia es tal que, en 1801, Alexander Von Humboldt, en su paso por Popayán, alaba sus determinaciones de longitud. Enterado José Celestino Mutis de la existencia de Caldas, lo vincula a la Expedición Botánica y decide construir un observatorio astronómico en los terrenos anexos a la Casa de la Expedición.

\section{Correspondencia:}

J. Gregorio Portilla, jgportillab@unal.edu.co

Recibido: 16 de enero de 2017

Aceptado: 16 de marzo de 2017 
Para ese entonces Caldas está más inclinado a la botánica, la que cultiva con gran pasión. Para finales de 1805 , a su regreso a Santafé y proveniente de la provincia de Quito, se le encarga la parte astronómica de la expedición — que él acepta un tanto a regañadientes (ver Caldas, 2016, carta 127, pág. 286) - para lo cual hará del observatorio su lugar exclusivo de trabajo.

Allí realiza observaciones astronómicas tendientes a determinar la posición geográfica de la edificación así como numerosas medidas meteorológicas. Su inclinación al periodismo científico $\mathrm{y}$, luego, al periodismo político como también su acercamiento al grupo de criollos con propósitos emancipadores lo hace descuidar casi que por entero sus labores científicas. Las diferencias entre criollos por la manera de conducir al país y la intención de reconquista por parte del imperio español obligó a Caldas a practicar la ingeniería militar. Habiendo sido exitoso, en sus inicios, el proceso de reconquista, Caldas es capturado y fusilado a finales de 1816.

La historiografía ordinaria se refiere a Caldas como astrónomo, botánico, meteorólogo, geógrafo, periodista e ingeniero militar. Es lugar común que tales actividades las realizó con tal ahínco y profundidad que era inevitable agregarle a la postre el mote de "sabio". Sin embargo, si se examinan con cuidado sus trabajos científicos publicados se encuentra que, en lo que se refiere a la astronomía, no hay ningún artículo o escrito que se dedique por entero a tal área del conocimiento. Varias memorias que, se sabe por sus cartas, redactó o estaba en proceso de culminar sobre aspectos netamente astronómicos, no lograron sobrevivir a los tumultuosos sucesos de las guerras de independencia o, cuanto menos, se encuentran perdidas. Además se infiere, por el poco material que sobrevivió, que sus conocimientos astronómicos eran básicos y que, a causa de su escaso dominio de las matemáticas, sus conocimientos de la mecánica celeste (para la época, el área de la astronomía que se encontraba en un notable desarrollo) eran, a lo sumo, rudimentarios.

El asunto de fondo es que Caldas no veía con buenos ojos la práctica de la astronomía per se, esto es, el estudio de los cuerpos que integran el universo con propósitos de conocer su origen, evolución, constitución, movimiento, etc. En su lugar, Caldas contempla la astronomía como una herramienta subsidiaria de la geografía (modernamente diríamos tanto de la geodesia como de la topografía). Una muestra clara de esa visión es esta: “Observar el cielo por observarlo, sería una ocupación honesta; pero no pasaría de una curiosidad estéril que llenase los momentos del hombre ocioso y acomodado. Este observador sería inútil, y la Patria lo miraría como un consumidor de quien no esperaba nada. Nosotros no queremos representar este papel en la sociedad: queremos que nuestros trabajos astronómicos mejoren nuestra geografía, nuestros caminos y nuestro Comercio" (Caldas, 1966, p. 183).

$\mathrm{Y}$ esa visión utilitarista de la astronomía se ve reflejada en casi todos los trabajos en los que Caldas aborda la observación de un cuerpo celeste. Los eclipses de Luna y de
Sol, las inmersiones y emersiones de los satélites del planeta Júpiter, los pasos del Sol y de estrellas por el meridiano del observador y el paso de Mercurio por en frente del disco solar tienen todos un común denominador: sirven para ubicar la posición geográfica del observador. Sólo se conoce una excepción: la observación, más o menos detallada, de un cuerpo celeste, la cual incluye la determinación de sus coordenadas celestes junto con la descripción morfológica del mismo. Tal objeto es el gran cometa de 1807.

El propósito de esta comunicación es, entonces, estudiar la observación que realizó Caldas del gran cometa de 1807 para que, de ese modo, se puedan inferir algunos aspectos de la experticia de Caldas en astronomía. En la primera sección describimos el descubrimiento y lo que conocemos de tal cometa; en la segunda se exponen las observaciones de Caldas; en la tercera sección se discuten las mismas y, finalmente, la última sección contiene las conclusiones.

\section{El cometa $\mathrm{C} / 1807 \mathrm{R} 1$}

Este cometa comenzó a ser observado en Europa en la segunda semana de septiembre de 1807 . Se atribuye la primera detección del objeto, el 9 de septiembre, a un monje de nombre Parisi (Russell, 1852) ubicado en la población siciliana de Castrogiovanni (actual Enna). Por su trayectoria, debió ser observado con anterioridad por numerosas personas situadas en el hemisferio sur (ver figura 1) pero no se han hallado registros al respecto. Parisi se le adelantó por casi dos semanas al gran astrónomo francés Jean-Louis Pons, quien lo observó el día 21. Pons, como se sabe, llegó a convertirse en el mayor descubridor de cometas de todos los tiempos (Hockey, 2007).

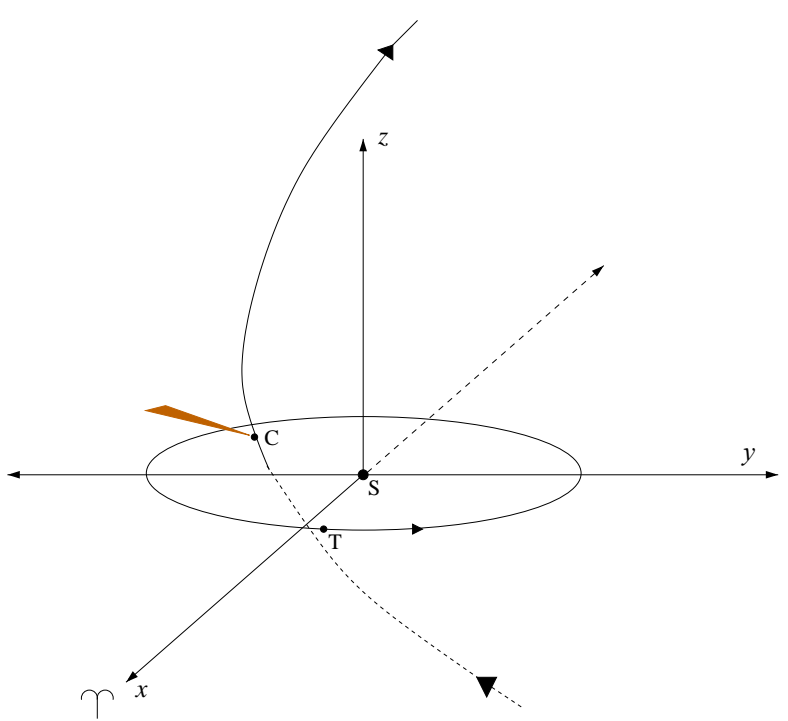

Figura 1. Posiciones del cometa (C) y de la Tierra (T) con respecto al Sol (S) en un sistema de coordenadas rectangular eclíptico heliocéntrico donde el eje $x$ apunta hacia el punto vernal $(\curlyvee)$ y el plano $x y$ es la eclíptica. Específicamente se muestran las posiciones relativas del cometa C/1807 R1 y la Tierra para el día 26 de septiembre de 1807. La curva muestra la trayectoria del cometa, segmentada (antes de pasar por la eclíptica) y continua (cuando la atravezó). 
Ese mismo día se reportaron observaciones hechas por Seth Pease desde los Estados Unidos así como de J. C. Thulis en Francia. El siguiente reporte se realizó cinco días después por parte de un profesor de matemáticas en la población de Vesoul, en Francia quien lo observó el 26.8 de septiembre; el día siguiente lo observaron también en Francia J. Vidal y H. Flaugergues. H. Pigott lo observó en Inglaterra el 28; H.S. Huth en Alemania el día siguiente y tanto Eule como González lo observaron desde Alemania y España, respectivamente, el día 30 (Kronk, 2003).

La órbita del cometa fue determinada por Bessel con base en cerca de 70 observaciones comprendidas entre el 22 de noviembre de 1807 y el 27 de marzo de 1808. En un principio asumió trayectoria parabólica, como era lo rutinario para las órbitas cometarias, pero la trayectoria resultante no satisfizo algunos puntos observacionales; al asumir órbita elíptica, tener en cuenta las perturbaciones de los planetas y aplicar el método de los mínimos cuadrados, recientemente inventado por Gauss, Bessel logró satisfacer todos los puntos observacionales. Esto es significativo, pues de ese modo el C/1807 R1 se convirtió en el segundo cometa, después del célebre cometa de Halley, que se demostró que era periódico. Los elementos orbitales del objeto, reportados por Bessel, se encuentran en la Tabla 1 (Kronk, 2003).

Tabla 1. Elementos orbitales del cometa C/1807 R1 determinados por Friedrich Bessel asumiendo órbita elíptica. Las coordenadas angulares están referidas al equinoccio medio y eclíptica del 2000.0.

\begin{tabular}{ll}
\hline Elemento & Valor \\
\hline Distancia pericéntrica $(q)$ & 0.646124 (u.a.) \\
Excentricidad $(e)$ & 0.995488 \\
Inclinación $(i)$ & $63.1762^{\circ}$ \\
Longitud del nodo ascendente $(\Omega)$ & $269.4837^{\circ}$ \\
Argumento de lat. del pericentro $(\omega)$ & $4.0970^{\circ}$ \\
Tiempo del paso por el pericentro $\left(t_{0}\right)$ & 19.2389 sep. 1807 \\
\hline
\end{tabular}

El semieje mayor $a$ del cometa en unidades astronómicas (u.a.) es, entonces:

$$
a=\frac{q}{1-e}=143.2012 \text { u.a., }
$$

y el periodo sideral $T$ dado por:

$$
T=\frac{2 \pi}{\sqrt{G M_{\odot}}} a^{3 / 2} \approx 1713 \text { años }
$$

siendo $\sqrt{G M_{\odot}}=0.01721$ u.a. ${ }^{3 / 2} \mathrm{~d}^{-1}$, donde $G$ es la constante de Cavendish $\left(G=6.673 \times 10^{-11} \mathrm{~m}^{3} \mathrm{~kg}^{-1} \mathrm{~s}^{-2}\right)$ y $M_{\odot}$ es la masa del Sol $\left(1.998 \times 10^{30} \mathrm{~kg}\right)$.

Por lo tanto, el cometa es de largo periodo y sólo retornará al sistema solar interior hasta alrededores del año 3520.

En cuanto a la morfología que presentaba el cometa, vale la pena mencionar la que aportó el astrónomo alemán Johann
Sigismund Huth quien, como ya se dijo, detectó el cometa el 29 de septiembre. Él registró que la cola del cometa "estaba situada casi en la dirección del ecuador... En el reflector el cuerpo del cometa aparecía como una bola perfecta del tamaño de Júpiter con solo una ligera coma”. También comentó que la cola estaba agudamente definida cerca de la coma, pero rápidamente difuminada y era sólo de unos $5^{\circ}$ de largo con un ancho de unos $0.5^{\circ}$ en su extremo (Kronk, 2003).

El cometa pudo seguir observándose a simple vista durante los meses de octubre y noviembre y algunos observadores alcanzaron a verlo sin ayuda de instrumentos ópticos hasta mediados del mes de diciembre. Huth reportó que a comienzos del mes de octubre la cola se dividió en dos componentes: una, de forma recta, con un largo de unos $6^{\circ}$ y la otra curvada no tan larga como la primera. Otro astrónomo alemán, Heinrich Olbers, notó, para la tercera semana de octubre, que la componente recta llegó a alcanzar $10^{\circ}$ mientras que la componente curvada era más corta, ancha y brillante con una longitud de unos $4.5^{\circ}$.

\section{Caldas observa el cometa}

Sólo se conocen tres fuentes en las que se menciona que Caldas observó el cometa C/1807 R1. La primera es el "número cero" del Semanario del Nuevo Reyno de Granada en la que el editor da a conocer la "interesante y curiosa" noticia de la aparición del cometa en los cielos vespertinos de la ciudad. En tal emisión, y previo a esta noticia, el editor solicita el permiso formal para la impresión del Semanario y describe los temas y contenidos que tendrá tal revista. La elaboración aparece con fecha del 4 de octubre de 1807. Pero, tan solo hasta el 3 de enero del siguiente año aparecería publicado el primer número del Semanario con un trabajo de Caldas titulado "Estado de la Geografía del Virreinato de Santafé de Bogotá con relación a la economía y al comercio" (ver anexo 1 de Nieto (2007)).

El "número cero" es relativamente poco conocido no siendo incluido en trabajos que, supuestamente, tienen por objeto dar a conocer el contenido total de los artículos del Semanario (Caldas, 1942) o sus obras completas (Caldas, 1966). Transcribimos a continuación la noticia del cometa tal y como apareció (con las modificaciones ortográficas pertinentes):

Pareciéndole al Editor interesante y curiosa la siguiente noticia, ha querido publicarla antes de dar principio a su Semanario.

Desde el 23 de setiembre se advirtió al fin de la constelación de la Virgen un cometa, y se comenzó a observar el 25 en el Observatorio astronómico de esta Capital. El encargado de este establecimiento D. Francisco Joseph de Caldas ha comenzado una serie de observaciones, y el 26 fijó el lugar del nuevo astro para 6 hor. 37 min. 17 seg. tiempo verdadero del meridiano del Observatorio: el resultado fue

Declinación austral........ 1 grad. $53 \mathrm{~min} .12 \mathrm{seg}$. Ascensión recta........... 217 grad. $57 \mathrm{~min} .00 \mathrm{seg}$. Longitud geocént..... 7 sig. 6 grad 19 min. 59 seg. Latitud boreal................ 12 grad. 25 min. $25 \mathrm{seg}$. 
El cuerpo del cometa pareció bastante terminado, y del diámetro de Marte poco más o menos. Su luz era rojiza, débil, y como dentro de nieblas. El núcleo estaba rodeado de una atmósfera luminosa prolongada en abanico hacia la parte opuesta al Sol, y cuya fuerza disminuía por grados insensibles hasta desaparecer. Esta ráfaga de luz, que puede llamarse barba por preceder al cometa en su curso era oblicua, y seguía la dirección de la Eclíptica; en fin su longitud pareció de 38 a 40 minutos.

Las observaciones que se han hecho, y que siguen haciéndose en el Observatorio, nos enseñarán el curso, distancia, órbita, etc. de este bello cometa. D. Francisco Joseph de Caldas se propone seguirle hasta su desaparición, e informar al público del resultado de sus trabajos. Mucho ha sentido este observador no haberlo advertido antes pero procurará llenar este vacío con las numerosas observaciones que actualmente ejecuta ${ }^{1}$.

La segunda fuente la constituye una de sus cartas dirigidas a Santiago Arroyo. Éste vivía para la época en Popayán y se constituyó, para Caldas, en su confidente científico y personal más cercano. En carta del 6 de noviembre de 1807, Caldas le escribe, entre otras cosas:

... Es verdad que este astro ha llenado mis momentos ha más de un mes, y creo no le dejaré hasta su total desaparición. El tiempo falta para calcular todos lo elementos de mis observaciones; pero las verá con el tiempo. Desde su aparición ha disminuido constantemente de diámetro y de luz. La cola que estaba en la inclinación de la eclíptica el 23 de septiembre, está hoy al contrario y vuelta al Norte. El abanico que formaba se ha dilatado más; esta es la forma que hoy tiene. Su curso es bien caprichoso; se vio en Virgo, cortó el ecuador entre el 28 y 29 de septiembre, pasó al Monte Menelao, tocó en la cabeza del Serpentario, y está ahora sobre el dorso de Hércules. Quién sabe dónde desaparecerá!... (carta 115, pág. 264, de Caldas (1978) ${ }^{2}$ ).

Una corta mención del cometa está contenida en la carta del 6 de febrero de 1808, también dirigida a Santiago Arroyo, en la que menciona que:

...Mi cabeza padeció con el cometa y ya estoy restableciéndome... (carta 120, pág. 279 de Caldas, 2016).

La tercera fuente la constituye el número 32 del Redactor Americano del Nuevo Reyno de Granada ${ }^{3}$ con fecha del 19 de marzo de 1808. Para ese entonces, y como ya vimos, el cometa ya había desaparecido por completo y había dejado de ser noticia. Sin embargo, el editor consideró incluirlo como novedad en razón a que ya disponía de información proveniente del extranjero con relación al cometa. La nota comprende dos partes. La primera consiste en reproducir, al pié de la letra, la nota ya publicada en el "número cero" del $\mathrm{Se}$ manario con excepción tanto de la nota introductoria como del último párrafo; sin embargo, no se alude a que tal texto ya había aparecido en otro periódico ni contiene información adicional de las observaciones que realizó Caldas. La segunda parte contiene noticias de observación del cometa en observatorios europeos, pero se da a entender que tal comunicación es una publicación proveniente de Filadelfia con fecha del 29 de diciembre de 1807. Como pié de página hay un dato interesante por parte del redactor: me consta que sin instrumento alguno, y sólo con la simple vista lo percibió desde el diez de septiembre un sujeto de esta ciudad de Santafé, y también otro en la de Cartagena, según carta remitida de allí.

\section{Discusión}

Si bien es cierto que Caldas no es el primero en reportar la observación de un cometa desde el virreinato de la Nueva Granada (véase Moreno (2005) para una descripción de observaciones anteriores), sí es el primero en reportar las coordenadas de un cometa para un instante de tiempo dado lo suficientemente precisas como para ser tenidas en cuenta en un proceso de determinación orbital tal y como se hacía a inicios del siglo XIX.

Lamentablemente, y en contradicción con lo que prometió tanto a sus lectores en el "número cero" del Semanario como con Arroyo, Caldas nunca publicó, comunicó o hizo mención del cúmulo de observaciones subsecuentes que realizó del cometa C/1807 R1 a lo largo de septiembre hasta, probablemente, noviembre de 1807. Algo parecido a un diario o libreta de apuntes donde anotó tales observaciones no se ha encontrado y lo más probable es que haya desaparecido para siempre. De modo tal que, para una discusión rigurosa de sus observaciones, sólo contamos con las coordenadas arriba registradas.

Comparando la información contenida en ambas fuentes se evidencia que Caldas, en efecto, comienza a observar el cometa a partir del 23 de septiembre (desde quien sabe qué lugar); sin embargo, se deduce que sólo desde el 25 de septiembre Caldas lo comienza a observar propiamente desde el Observatorio y que sólo hasta el día siguiente (26), contando con la instrumentación adecuada, puede determinar su ubicación mediante coordenadas ecuatoriales. Coincidencialmente, ese día (o más exactamente el 26.81 TU) es cuando el cometa estuvo más cerca de la Tierra a una distancia de 1.1533 u.a. Por la fecha de observación se infiere que Caldas merece ser incluido entre los primeros observadores de dicho cometa, al igual que se constituye en el más meridional de todos los observadores que registró sus coordenadas.

El cometa era visible desde Santafé unos 20 a 30 minutos después de la puesta de Sol desde los primeros días de septiembre (contando con las propicias condiciones climatológicas). De haber existido alguien a la alerta, una especie

\footnotetext{
${ }^{1}$ http://www.banrepcultural.org/blaavirtual/historia/semanario/indice.htm (accesado el 5 de diciembre de 2016).

${ }^{2}$ Para esta carta no se ha referenciado el libro más actualizado (Caldas, 2016) en razón de que tal versión presenta, en dicha carta, algunos errores de digitación que cambian ligeramente el sentido de la misma.

${ }^{3}$ http://www.banrepcultural.org/blaavirtual/hemeroteca-digital-historica/el-redactor-americano-periodico-del-nuevo-reyno-de-granada (accesado el 5 de diciembre de 2016).
} 
de "centinela del cielo" (que Caldas no lo era), era bien posible distinguirlo desde Santafé en tales fechas muy cerca del horizonte occidental. Recuérdese que alguien alcanzó a observarlo desde Santafé desde el 10 de septiembre, apenas un día después de la primera observación reportada por alguien en el mundo. Agravando el asunto, todo parece indicar que la sociedad santafereña de ese tiempo no es que fuera muy proclive a la observación del cielo. El conocido diario que llevaba Caballero (1946) de comienzos del siglo XIX, en el que registraba diversidad de aspectos de la sociedad de su tiempo (incluyendo la anotación de algunos fenómenos naturales) no se refiere para nada en absoluto al cometa.

Caldas establece el tiempo de la observación del 26 de septiembre en tiempo solar verdadero, que corresponde al ángulo horario del Sol en ese instante, esto es, $H_{\odot}=6^{h} 37^{m} 17^{s}$. Con ayuda de un programa de computador hemos reproducido el cielo en esa noche ${ }^{4}$. Se reprodujo la bóveda celeste tal y como estaba para un observador ubicado en unas coordenadas de latitud y longitud, respectivamente, de $\phi=4^{\circ} 35^{\prime} 46^{\prime \prime}$ y $\lambda=74^{\circ} 04^{\prime} 39^{\prime \prime}$ (coordenadas del el Observatorio respecto al elipsoide WGS84) de modo tal que el Sol tuviese un valor de ángulo horario de $6^{h} 37^{m} 17^{s}$. La ascensión recta del Sol (referida al equinoccio y eclíptica media de la fecha) era de $\alpha_{\odot}=12^{h} 10^{m} 43^{s}$ de lo que se infiere inmediatamente que el tiempo sideral local era igual a $H_{\curlyvee}=18^{h} 48^{m} 0^{s}$. Para tal fecha la ecuación del tiempo es de $8^{m} 36^{s}$, así que el tiempo solar medio, para el meridiano del Observatorio Astronómico, fue de $18^{h} 28^{m} 41^{s}$. Una descripción de una porción de la esfera celeste como se veía para un observador en Bogotá puede verse en la figura 2.

Es curioso que Caldas, al ubicar las coordenadas del cometa, haya hecho la observación aun cuando el cielo no estaba ennegrecido por completo, pues en ese instante apenas el Sol tenía una altura de $-9^{\circ} 23^{\prime}$. Para el instante en cuestión las coordenadas horizontales (azimut y altura) del cometa eran: $A \approx 265^{\circ} 39^{\prime}, h \approx 25^{\circ} 33^{\prime}$, lo que quiere decir que el cometa aun estaba lo suficientemente alto sobre el horizonte como para ser observado adecuadamente al menos por otros 45 minutos. Probablemente Caldas consideró que más tarde se podría malograr la observación. Ignoramos el tipo de instrumentación con la que hizo la observación pero, probablemente, fue un refractor pequeño de la casa Dollond que, se sabe, habían al menos dos en el Observatorio por aquellos años. Caldas debió contrastar la observación con respecto a un atlas celeste para que, de ese modo, estimara las coordenadas ecuatoriales. Ignoramos cual atlas pudo haber sido. En los varios inventarios de la biblioteca de Caldas que se encuentran en Hernández de Alba (1986) (y un tanto más simplificado en Díaz (1997)) no aparecen registrados atlas celestes, lo que dice muy poco en realidad, ya que Caldas pudo llevarlos consigo cuando salió de Santafé o fueron sujetos a la destrucción o al robo cuando ingresaron violentamente las tropas de Bolívar al Observatorio en la toma de la ciudad en diciembre de 1814.

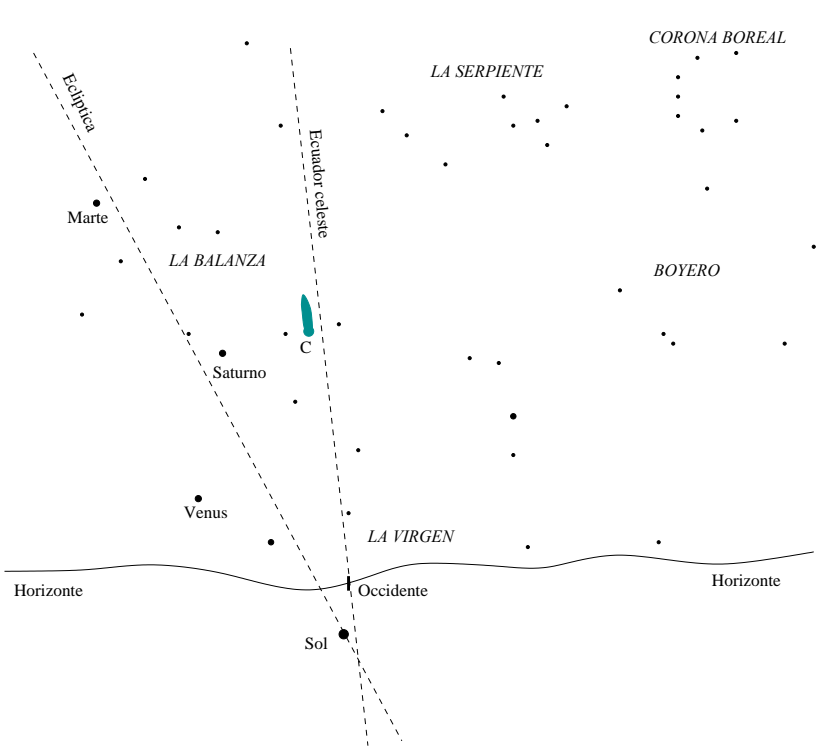

Figura 2. Posición del cometa (C ) en el horizonte occidental para un observador ubicado en Santafé de Bogotá a las $18^{h} 28^{m} 41^{s}$ (tiempo solar medio referido al meridiano del Observatorio Astronómico) del día 26 de septiembre de 1807. El horizonte, que se ha descrito como una curva continua ondulada, no representa el horizonte real.

Pero tales cartas celestes bien pudieron haber sido o el atlas celeste de Flamsteed (Fortin, 1776) o el Uranographia de Bode (1801) o incluso ambos. Humboldt, en su estadía en Quito, le recomendó a Caldas la adquisición del primero (ver pág. 259 de Díaz (1997)) y es muy posible que así lo hiciera. Y, de haber sido el Uranographia, Caldas contaba entonces con uno de los mejores atlas con que los astrónomos podían disponer en aquel tiempo.

En la tabla 2 están registradas, en notación moderna, las coordenadas ecuatoriales y eclípticas que dedujo Caldas cuando ubicó la posición del cometa en sus cartas. Simplemente como aclaración, la longitud eclíptica, como la reportó Caldas en el "número cero" del Semanario, aparece, como ya vimos, como: 7 sig. 6 grad 19 min. 59 seg. Quiere decir que el cometa se encontraba a una longitud de $7 \times 30^{\circ}+6^{\circ} 19^{\prime} 59^{\prime \prime}=$ $216^{\circ} 19^{\prime} 59^{\prime \prime}$ ya que cada signo zodiacal (contado a partir del punto vernal y sobre la eclíptica) se asumía con una extensión de $30^{\circ}$.

Algo que puede desprenderse de los valores que calculó Caldas es que, al ser extraídas de las cuadrículas de un atlas (como cualquiera de los dos citados arriba) no pueden aparecer al segundo de arco (como lo escribe Caldas) pues no es posible derivar las coordenadas a ese nivel de detalle a partir de las mismas; si acaso, a fracción de minuto de arco. Adicionalmente, todo parece indicar que tanto el par ecuatorial (ascensión recta $(\alpha)$, declinación $(\delta)$ ) como el par eclíptico (longitud eclíptica $(\lambda)$, latitud eclíptica $(\beta)$ ) los dedujo cada uno por aparte. Tal procedimiento se puede hacer sin mayor

\footnotetext{
${ }^{4}$ Se ha utilizado, para tal efecto, el programa Voyager 4 de la empresa Carina Software.
} 
inconveniente, dado que los atlas en aquellos años disponían de cuadrículas apropiadas tanto sobre el ecuador celeste como sobre la eclíptica.

Tabla 2. Coordenadas ecuatoriales y eclípticas reportadas por F. J. Caldas en el instante 6 hor. 37 min. 17 seg. tiempo verdadero del meridiano del Observatorio del 26 de septiembre de 1807.

\begin{tabular}{cc}
\hline Coordenadas & Valor \\
\hline$\delta$ & $-01^{\circ} 53^{\prime} 12^{\prime \prime}$ \\
$\alpha$ & $14^{h} 31^{m} 48^{s}$ \\
$\lambda$ & $216^{\circ} 19^{\prime} 59^{\prime \prime}$ \\
$\beta$ & $12^{\circ} 25^{\prime} 25^{\prime \prime}$ \\
\hline
\end{tabular}

Ello explica porque, desde el punto de vista de alguien familiarizado con la astronomía de posición, los valores de la tabla 2 difieren, digamos, de las coordenadas eclípticas que se podrían calcular a partir de las coordenadas ecuatoriales ${ }^{5} \mathrm{o}$ al revés. Como es bien sabido, bastaría con extraer un solo par coordenado a partir del atlas y calcular, con las ecuaciones de la trigonometría esférica, el otro par. Pero Caldas opta por no hacerlo así lo que a la postre genera valores que no son equivalentes. A manera de ejemplo, si se desea calcular la longitud eclíptica a partir de los valores de ascensión recta y la declinación dados por Caldas y utilizar la expresión (Portilla, 2009):

$$
\lambda=\tan ^{-1}\left(\frac{\operatorname{sen} \delta \operatorname{sen} \varepsilon+\cos \delta \cos \varepsilon \operatorname{sen} \alpha}{\cos \alpha \cos \delta}\right),
$$

es preciso, para tener el valor de $\lambda$ que reporta Caldas, utilizar un valor de $\varepsilon=22^{\circ} 43^{\prime} 45^{\prime \prime}$, el cual es demasiado bajo. En cambio, si se utiliza el valor de $\varepsilon=23^{\circ} 27^{\prime} 53^{\prime \prime}$ se obtiene un valor de $\lambda=216^{\circ} 12^{\prime} 18^{\prime \prime}$, el cual es unos $7.5^{\prime}$ más bajo que el que obtiene Caldas.

Al dejar al margen aspectos tales como la incertidumbre en el uso apropiado de las escalas de tiempo así como el desconocimiento de la fecha del equinoccio de referencia del atlas que usó Caldas, al introducir los elementos orbitales del cometa contenidos en la tabla 1 en el programa Voyager 4 y reproducir las coordenadas ecuatoriales del objeto correspondientes al instante 6 hor. $37 \mathrm{~min} .17 \mathrm{seg}$. del 26 de septiembre de 1807 de tiempo verdadero del meridiano del Observatorio, tal programa arroja lo siguiente: $\alpha=14^{h} 31^{\prime} 21^{\prime \prime}, \delta=$ $-01^{\circ} 55^{\prime} 38^{\prime \prime}$ (equinoccio instantáneo de la fecha), lo que indica un error cercano a los 6 minutos de arco en ascensión recta y menos de dos minutos en declinación.

Con relación a la información contenida en la carta del 6 de noviembre dirigida a Arroyo, lo que narra Caldas sobre la ubicación del cometa a finales de septiembre y hasta el 6 de noviembre se corresponde con los hechos. En efecto, es posible constatar a través del programa Voyager 4 que la cola del cometa, para la noche del 23 de septiembre, tiene un ángulo pequeño con relación a la dirección de la eclíptica (asumiendo que la cola está en una dirección opuesta al Sol). El paso por el ecuador celeste se verificó el 28 de septiembre, aproximadamente a las $18^{h} 23^{m}$ tiempo local medio con relación al meridiano del Observatorio. Para inicios del mes de octubre, el cometa comienza a aumentar en declinación. El comentario de que pasó por el "Monte Menelao” se explica en razón a que algunos autores entendían como una pequeña constelación con tal nombre a un sector en lo que modernamente constituyen regiones de la constelación del Boyero y la Serpiente; con el tiempo dicha constelación pasó a desuso. El hecho de que el Uranographia registre tal constelación ${ }^{6}$ apoya la tesis de que Caldas contara con uno de tales ejemplares. En la figura 3 se puede observar la trayectoria del cometa calculada por el programa Voyager 4 entre las fechas que comentó Caldas en su carta del 6 de noviembre a Arroyo.

Si hemos de creer en el testimionio de Caldas, y nada nos hace pensar de lo contrario, él terminó reuniendo un número importante de observaciones astronómicas del cometa entre finales de septiembre y al menos hasta inicios de noviembre; ello le demandó un gran esfuerzo que incluso llegó a pagar con detrimento de su salud. Sin embargo, tenemos serias dudas de que Caldas hubiése tenido los conocimientos necesarios para determinar la órbita del cometa. Nada en los escritos que le sobreviven indican un dominio de la mecánica celeste que le hubieran permitido obtener los parámetros orbitales y, de ese modo, conocer la forma de la órbita. Lo cierto es que cuando tuvo a su entera disposición el Semanario (a inicios de 1809) y bien podía escribir allí lo que quisiera, nunca publicó el resultado de tales observaciones.

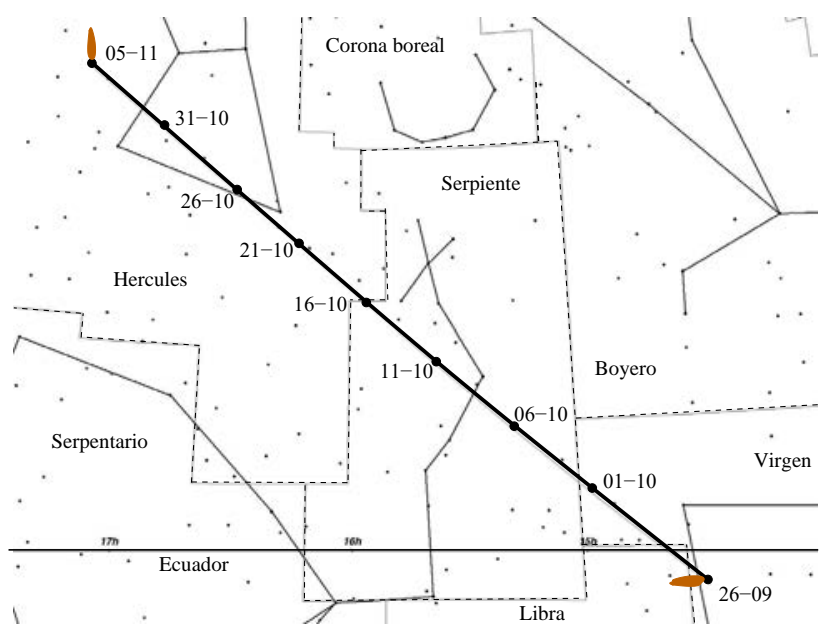

Figura 3. Trayectoria del cometa C/1807 R1 (curva en negrilla) desde el 26 de septiembre hasta el 5 de noviembre de 1807. Solo se muestra la dirección de la cola (opuesta al Sol) en tales fechas extremas. Los límites de las constelaciones son los actualmente utilizados. La longitud asignada para la cola es sólo por propósitos ilustrativos y no reflejan la verdadera magnitud reportada por algunos observadores europeos.

\footnotetext{
${ }^{5}$ Tomando un valor específico de la oblicuidad de la eclíptica, que para 1807 era de $\varepsilon=23^{\circ} 27^{\prime} 53^{\prime \prime}$ (Farrar, 1827).)

${ }^{6} \mathrm{El}$ atlas de Flamsteed no la registra.
} 
El hecho de que en el "número cero" del Semanario haya prometido a sus lectores nuevas observaciones así como el conocimiento de la órbita sugiere que tales palabras son más bien una reacción precipitada y emotiva frente a su descuido en no haber sido el primero en advertir el cometa. Solo de ese modo se explica que Caldas, a manera de ofrecimiento de disculpas, mencione que "mucho ha sentido no haberlo advertido antes pero procurará llenar este vacío con las numerosas observaciones que actualmente ejecuta". Para una persona quien era conocida en la ciudad como "el astrónomo" (ver, por ejemplo, la carta 151, pág. 322 de Caldas, 2016) y dado que era bien consciente de que habían santafereños que lo veían con recelo y envidia (ver carta 107, pág. 263 de Caldas, 2016), pensó que la promesa de un enorme cúmulo de observaciones en el futuro era la mejor manera de resarcirse, al menos hasta que, como es lo usual, las cosas pasaran al olvido.

En cuanto a la forma del cometa, Caldas comenta que su diámetro es del de "Marte poco más o menos". Probablemente le pareció que en brillo fueran semejantes ya que la magnitud del planeta rojo era en ese momento de 1.2 y observadores en Europa estimaron la magnitud del cometa para finales de septiembre entre 1 y 2. Caldas observa que el cuerpo del cometa pareció bastante terminado, lo que está de acuerdo con la observación de varios astrónomos europeos. Por lo demás, Caldas menciona, en el "número cero" del Semanario, el reporte usual típico de un cometa, describiendo su coma y la cola (aunque no utilizó tales términos) la que describe en dirección hacia la eclíptica y contraria a la del Sol. A la cola del cometa la describe como una "ráfaga de luz, que puede llamarse barba por preceder al cometa en su curso". Quiere decir con ello que la cola del cometa está en una dirección tal que va precediendo (aunque no exactamente) el camino que va describiendo sobre la bóveda celeste.

A manera de paréntesis, sería además interesante examinar el original de la carta dirigida a Arroyo del 6 de noviembre. Las palabras: "El abanico que formaba se ha dilatado más; esta es la forma que hoy tiene.” hacen pensar que en la margen de las mismas se encuentre un dibujo que describa la forma de la cola y que en la obras que han reproducido las cartas conocidas de Caldas no lo han incluido. También es posible que lo que quiso decir es que la forma de la cola era en "abanico".

Ahora bien, lo que no se entiende bien es el valor que Caldas asigna a la longitud de la cola: de 38 a 40 minutos $\left(\sim 0.7^{\circ}\right)$. Bien es cierto que en ese "número cero" no utiliza el término cola como tal, pues en su lugar utiliza "atmósfera luminosa prolongada en abanico" así como también "ráfaga de luz" y "barba”. Tampoco utiliza el término “coma”. Pero, si se refiere a ésta, sería un valor desproporcionadamente alto para su tamaño. Así que lo más probable es que se refiera, en efecto, a la cola. Pero, entonces, cabe la pregunta: ¿por qué asignó un tamaño tan corto a la extensión de la cola? Se sabe que para finales del mes de septiembre varios observadores europeos estimaron una longitud de la cola que es unas diez veces más grande que lo que reporta Caldas. Como sólo se dispone de ese dato observacional del 26 de septiembre y, como comentamos atrás, tal observación se hizo todavía con luz crepuscular, se infiere que es posible que tuviera dificultades en observar adecuadamente la verdadera la longitud del cometa por falta de contraste. Si esto es cierto, se deduce que no pudo observar el cometa pocos minutos después, pues eso le hubiera permitido observar la verdadera longitud de la cola. Por demás, la falta de constraste no pudo deberse a la luz proveniente de la Luna, ya que para el momento en cuestión ésta se encontraba por debajo del horizonte con una altura cercana a los $-60^{\circ}$.

El reporte que hace Caldas de una cola tan corta para el cometa es inusual. Eso, aunado a que el objeto sólo se percibiera en Santafé hasta el 23 de septiembre (exceptuando la observación que se reportó el 10 de septiembre) cuando, al menos teóricamente, era posible observarlo desde varios días antes, sugiere algún tipo de dificultad en la observación, al menos hacia el horizonte occidental. Los observadores en Europa en esa última semana de septiembre atestiguan una observación de la cola del cometa que, por su extensión y tamaño, muy difícilmente podía pasar desapercibida.

Igualmente diciente es que Caldas, en su carta a Arroyo, en la que sí habla de "cola" propiamente, no menciona la división de la misma en dos. Eso, como vimos, comenzó a observarse en Europa desde inicios del mes de octubre; pero Caldas, que desde finales del mes de septiembre lo observó — presumiblemente- de forma continua hasta al menos el 6 de noviembre, no comenta nada en absoluto con relación a tal hecho. De haber Caldas observado tal fenómeno, por su espectacularidad y relativa rareza, muy difícilmente lo hubiera dejado pasar por alto en comunicación con su amigo.

Todos estos detalles implican dificultad en la observación de la cola en todo su esplendor, lo que puede sugerir la ocurrencia de algún fenómeno que estaba sucediendo en el cielo en los atardeceres santafereños que estorbaba e impedía la adecuada observación del objeto. Algo semejante a una nube de polvo (aerosol estratosférico), bruma recurrente o el humo proveniente de incendios forestales masivos.

La posibilidad de la existencia de una erupción volcánica que dé lugar a un brillo crepuscular (twilight glow) que dificulte la observación astronómica no puede descartarse dado que un fenómeno de tal naturaleza se dio en algún momento de diciembre de 1808 (Guevara-Murua, 2014). A tal suceso se le denomina la "erupción desconocida", pues se ignora con exactitud cuando y en donde ocurrió. El mismo Caldas reportó en el Semanario (pág. 355 de Caldas (1966)) los efectos de esa supuesta erupción. En efecto, allí da a conocer la existencia, para mediados de diciembre de 1808, de una anomalía en la observación del Sol sobre el horizonte, principalmente en el poniente, consistente en una fuerte atenuación de la luz solar $^{7}$; así mismo reportó la imposibilidad de observar estrellas de cuarta y quinta magnitud. Es posible que un fenómeno semejante, pero de menor intensidad, hubiese

\footnotetext{
${ }^{7}$ Caballero (1946) no registra nada de esto en su diario aunque se sabe, por el mismo Caldas, que causó no poca inquietud en unos cuantos santafereños.
} 
tenido lugar unos quince meses antes, lo que podría explicar varios aspectos inusuales de la observación del cometa realizada por Caldas.

Una opción más probable es que aquello que impidió la observación adecuada del cometa sea el conjunto de nubes, al parecer perennes, que se formaban encima del lugar del Salto del Tequendama. Tal cascada está ubicada en el municipio de Soacha, Cundinamarca, y se forma por la caída abrupta de unos 150 metros del cauce del río Bogotá. El salto, ubicado a unos $24 \mathrm{~km}$ en línea recta del centro de Bogotá, posee un azimut, visto desde el Observatorio Astronómico, de unos $264^{\circ}$. Una fuente relata cómo era de intensa la formación de las nubes sobre el salto hace dos siglos. Humboldt (1982), que estuvo en Santafé en 1801, escribe que visto desde allí "el punto donde está situado el salto se distingue claramente sobre el horizonte por las espesas nubes que lo cubren constantemente" (pág. 52,a); y más adelante: "Yo he visto cascadas más ricas en agua y sin embargo, nunca observé sobre ninguna un nubarrón tan permanente y espeso sobre el Tequendama" (pág. 68,a).

Entre mediados de septiembre hasta mediados de octubre, las fechas más propicias para observar con mayor esplendor el cometa, el azimut del objeto, visto desde el Observatorio Astronómico, osciló desde un valor de $250^{\circ}$ hasta $280^{\circ}$, esto es, una o dos decenas de grados alrededor del punto donde se ubica el Salto del Tequendama. Si existió, durante ese intervalo de tiempo, una acumulación de nubes sobre la región del salto, en particular del tipo cirrus a alturas superiores a los $6 \mathrm{~km}$, para observadores en Santafé el espectáculo del cometa con su cola alargada resultaba particularmente atenuado. Lamentablemente, la no existencia de reportes de observadores en otras zonas del virreinato no permiten arrojar mayor luz sobre el asunto.

\section{Conclusiones}

El reporte que hace Caldas del cometa C/1807 R1 así como una fijación de sus coordenadas celestes realizada el 26 de septiembre de 1807 bien merece ser incluido como uno de los primeros sobre la observación de ese objeto. Es evidente que el relativo desconocimiento del "número cero" del Semanario entre los historiadores y astrónomos colombianos de los dos siglos anteriores condujo a que referencias obligadas sobre historiografía cometaria tales como la de Kronk (2003) ignoren la observación de Caldas, la que, de paso, se constituye en el reporte más meridional de tal objeto.

La principal observación del cometa C/1807 R1 que realizó Caldas, la del día 26 de septiembre, la hizo todavía con luz crepuscular y coincidió con el momento de máximo acercamiento a la Tierra. Fijó, con relativamente buen tino, las coordenadas ecuatoriales en un atlas celeste. Pero lo hizo también, de forma independiente, con las coordenadas eclípticas. Sin embargo, no se molestó en verificar la equivalencia entre las coordenadas ecuatoriales y eclípticas. Se ignora si era consciente de que tal cálculo se podía hacer y si era de su dominio. Además expresó tales coordenadas al segundo de arco, lo que no es posible hacerlo contando con los atlas de que disponía. En tal sentido, es muy posible que el atlas que usó haya sido el Uranographia de Bode.

El reporte que hace Caldas del desplazamiento del cometa sobre la bóveda celeste desde finales de septiembre hasta comienzos de noviembre es consistente con los elementos orbitales calculados por Bessel. En cuanto al reporte morfológico, se evidencia que Caldas no pudo observar la cola del cometa como lo vieron los astrónomos europeos. Para Caldas la cola tenía una longitud significativamente más corta y conservó su unidad. Esto sugiere la presencia de algún tipo de fenómeno que estorbó la observación. Aquí proponemos dos posibilidades: la primera es la presencia de un brillo crepuscular derivado de una erupción volcánica, lo que implica la existencia de una erupción de corta intensidad acaecida unos 15 meses antes de la denominada "erupción desconocida" de diciembre de 1808. La segunda, y más probable, es la existencia de nubes del tipo cirrus ubicadas a alturas superiores a los $6 \mathrm{~km}$ ubicadas sobre la región del Tequendama cuya presencia de nubes era muy notoria en tal zona dos siglos atrás. No puede descartarse, tampoco, la presencia de una bruma espesa presente en el periodo de tiempo de la observación del cometa.

\section{Agradecimientos}

El autor agradece al historiador Iván Felipe Suárez, Director de la Casa Museo Caldas, quien llamó la atención del autor sobre la existencia del "número cero" del Semanario. Igualmente, el autor agradece los cometarios de un árbitro anónimo.

\section{Conflicto de intereses}

El autor declara que no tiene ningún conflicto de intereses.

\section{Referencias}

Bode J. E. (1801). Uranographia Sive Astrorum Descriptio, Berlín.

Caballero J. M. (1946). Particularidades de Santafé, Biblioteca Popular de Cultura Colombiana, Bogotá.

Caldas F. J. (1942). Semanario del Nuevo Reino de Granada, vol. 1, Biblioteca Popular de Cultura Colombiana, Bogotá.

Caldas F. J. (1966). Obras Completas de Francisco José de Caldas, Universidad Nacional de Colombia, Imprenta Nacional, Bogotá.

Caldas F. J. (1978). Cartas de Caldas, Academia Colombiana de Ciencias Exactas, Físicas y Naturales \& Colciencias, Bogotá.

Caldas F. J. ( 2016). Cartas de Caldas Ilustradas, Universidad Distrital Francisco José de Caldas, Asociación de Amigos de la Casa Museo Caldas y Academia Colombiana de Ciencias Exactas, Físicas y Naturales, Bogotá.

Díaz S. (1997). Nueva Aproximación a Francisco José de Caldas, Academia Colombiana de Historia, Editora Guadalupe, Bogotá.

Farrar J. (1827). An Elementary Treatise on Astronomy, Hilliard Metcalf and Co., Cambridge. 
Fortin M. J. (1776). Atlas Céleste de Flamstéed, Approuve par l'Académie Royale des Sciences, F. G. Deschamps, París.

Guevara-Murua A., et al. (2014). Clim. Past., 10, 1707.

Hernández de Alba G. (1986). Historia Documental de la Real Expedición Botánica del Nuevo Reino de Granada después de la muerte de su director Don José Celestino Mutis 1808-1952, Fundación Segunda Expedición Botánica, Editora Guadalupe, Bogotá.

Hockey T. (2007). The Biographical Encyclopedia of Astronomers, Springer, Nueva York.

Humboldt A. (1982). Alexander Von Humboldt en Colombia. Extractos de sus diarios, Academia Colombiana de Ciencias Exactas, Físicas y Naturales \& Academia de Ciencias de la
República Democrática Alemana, Publicismo y Ediciones, Bogotá.

Kronk G. (2003). Cometography: A Catalog of Comets (Vol. 2: 1800-1899), Cambridge University Press, Cambridge.

Moreno F. (2005). El Astrolabio, 5, 18.

Nieto M. (2007). Orden natural y orden social: ciencia y política en el Semanario del Nuevo Reyno de Granada, Legis S. A., Bogotá.

Portilla J. G. (2009). Elementos de Astronomía de Posición, Editorial Universidad Nacional de Colombia, Bogotá.

Russell J. (1852). The Comets: A Descriptive Treatise Upon These Bodies, John W. Parker and Son, Londres. 